\title{
CARACTERIZACIÓN MORFOLÓGICA Y BIOQUÍMICA DE TIPOS DE GUAYABA (Psidium guajava L.) COLECTADOS EN SUMAPAZ, COLOMBIA
}

\author{
MORPHOLOGICAL AND BIOCHEMICAL CHARACTERIZATION OF GUAVA \\ (Psidium guajava L.) TYPES COLLECTED IN SUMAPAZ, COLOMBIA
}

\author{
Alba G. Fajardo-Ortíz' ${ }^{1}$, Juan P. Legaria-Solano ${ }^{1}$, \\ Jairo E. Granados-Moreno ${ }^{2}$, Juan Martínez-Solís ${ }^{1}$ y Álvaro Celis-Forero ${ }^{3}$
}

'Universidad Autónoma Chapingo, Departamento de Fitotecnia, Chapingo, Texcoco, Estado de México, México. ${ }^{2}$ Universidad de Cundinamarca, Facultad de Ciencias Agropecuarias, Cundinamarca, Colombia. ${ }^{3}$ Universidad de Cundinamarca, Dirección de Investigación, Cundinamarca, Colombia.

*Autor de correspondencia (legarias.juan@yahoo.com)

\section{RESUMEN}

La guayaba (Psidium guajava L.) es una especie de fruta neotropical. En Colombia su variabilidad morfológica y bioquímica ha sido poco estudiada. En esta investigación se caracterizaron frutos de seis localidades en árboles de guayabo silvestres de la provincia de Sumapaz, estado de Cundinamarca, Colombia con el objetivo de determinar su nivel de variación. Se registraron ocho caracteres cuantitativos y seis cualitativos, se descartaron los últimos por su bajo aporte en la variación de los genotipos. El promedio del peso del fruto $(78.16 \mathrm{~g})$, índice de redondez $(92 \%)$, sólidos solubles totales $\left(8.62^{\circ} \mathrm{Brix}\right)$ y contenido de vitamina $\mathrm{C}\left(170.82 \mathrm{mg} 100 \mathrm{~g} \mathrm{~g}^{-1}\right)$ indicaron que existe potencial en los genotipos silvestres estudiados para su utilización directa. Entre los sitios muestreados se encontró a la accesión de Guavio como sobresaliente por características morfológicas, con peso promedio de fruto de 120.17 $\mathrm{g}$, diámetro longitudinal y transversal de 7.48 y $5.92 \mathrm{~cm}$, respectivamente, aunque el contenido de SST ( $\left.6.46^{\circ} \mathrm{Brix}\right)$ o vitamina C $\left(158.85 \mathrm{mg} 100 \mathrm{~g} \mathrm{~g}^{-1}\right)$ no fue sobresaliente. Las colectas de los demás sitios mostraron valores desde 8.55 hasta $9.43^{\circ}$ Brix de sólidos solubles totales, mientras que en contenido de ácido ascórbico los genotipos de Udec mostraron el valor más alto (201.61 $\mathrm{mg} 100 \mathrm{~g}^{-1}$ ). En el análisis de agrupamiento por localidades se encontró que la accesión de Guavio conformó un solo grupo y el resto de las accesiones otro. El tipo Guavio no presentó valores notables para los caracteres bioquímicos, pero sí en las características morfológicas; la separación que mostró con respecto al resto de las colectas durante el agrupamiento está centrada en la influencia de las características del fruto. Los resultados indican la existencia de algunos tipos de guayaba silvestre de importancia para uso directo 0 mejoramiento genético en la provincia de Sumapaz, Colombia.

Palabras clave: Morfología, vitamina C, grados Brix, mejoramiento genético.

\section{SUMMARY}

Guava (Psidium guajava L.) is a neotropical fruit species. In Colombia, its morphological and biochemical variability has been poorly studied. In this research, fruits of wild guava from six localities of the Sumapaz province, state of Cundinamarca, Colombia were characterized. Eight quantitative and six qualitative characters were recorded in order to determine their level of variation, the latter were discarded due to low contribution to the variation of genotypes. The averages of fruit weight $(78.16 \mathrm{~g})$, roundness index (92 $\%)$, total soluble solids (8.62 ${ }^{\circ} \mathrm{Brix}$ ) and vitamin C content (170.82 mg 100 $\mathrm{g}^{-1}$ ) indicate that there is potential in the wild varieties studied for their direct utilization. Among the sampled sites, the accession from Guavio was found to be outstanding in morphological characteristics, with average fruit weight of $120.17 \mathrm{~g}$, longitudinal and transverse diameter of 7.48 and $5.92 \mathrm{~cm}$ respectively, but it did not stand out in traits such as total soluble solids (6.46

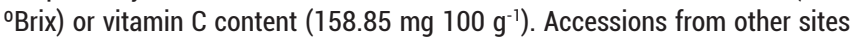
ranged from 8.55 to $9.43^{\circ} \mathrm{Brix}$ of total soluble solids while in ascorbic acid content the Udec genotypes showed the highest value $\left(201.61 \mathrm{mg} 100 \mathrm{~g}^{-1}\right)$ In the grouping analysis by locations, it was found that the Guavio accession formed a single group and the rest of the accessions another one. The Guavio type did not show outstanding values in the biochemical characters, but it did in morphological traits; the separation it showed in relation to the rest of the accessions is centered on the influence of fruit characteristics. Results indicate the existence of some types of wild guava of importance for direct use or genetic improvement in the province of Sumapaz, Colombia.

Index words: Morphology, vitamin C, Brix degrees, genetic improvement.

\section{INTRODUCCIÓN}

La familia Myrtaceae cuenta con numerosas especies comestibles, entre ellas la guayaba (Psidium guajava L.) (Jaiswal y Jaiswal, 2005). Esta especie es originaria de América, pero introducida a otras regiones tropicales y subtropicales del mundo donde se cultiva por lo atractivo del fruto y propiedades nutritivas como su alto contenido de ácido ascórbico, precursor de vitamina $\mathrm{C}$, vitamina $\mathrm{A}$, tiamina, riboflavina y minerales como calcio, hierro y fósforo. También se le han atribuido propiedades medicinales y utilizada en medicina tradicional en diferentes países del mundo (Rodríguez et al., 2010).

Esta especie fue semi-domesticada hace más de 2000 años y actualmente toma importancia por sus altos niveles deácido ascórbico al ocupar el tercer lugar en concentración después de la cereza de Barbados (Malpighia glabra L.) con más de 1000 mg y la de Angola (Emblica officinalis L.) con 500 a $700 \mathrm{mg} 100 \mathrm{~g}^{-1}$ de ácido ascórbico. A diferencia de la guayaba, estas frutas son ácidas, lo que limita su consumo, mientras que los frutos de la guayaba tienen 
la ventaja de consumirse en fresco o procesados (Mata y Rodríguez,1990). Por su contenido de fenoles totales se constituye en una fuente de antioxidantes naturales para la nutrición humana (Silva y Sirasa, 2018).

El guayabo se desarrolla bien desde los 0 hasta los 1500 msnm (Mata y Rodríguez 1990); otros autores han reportado adaptabilidad a 2000 msnm (Insuasty et al., 2014). Un ejemplo de los extremos es Hawaii, donde se le cultiva desde el nivel del mar hasta los 1200 msnm. En las dos condiciones el crecimiento y la producción total de fruta se reducen, aunque los cultivos más exitosos se obtienen entre los 150 y $450 \mathrm{msnm}$.

La producción mundial de guayaba es de aproximadamente 1.2 millones de toneladas, la India y Pakistán son los mayores productores (50\%), seguidos de México (25\%) y otros países como Colombia, Egipto y Brasil (Yam et al., 2010). En Colombia la producción se concentra principalmente en los estados de Santander y Boyacá (60 \%), Tolima (10 \%), Cundinamarca (9\%), Huila, Antioquia, Cauca, Nariño y Atlántico (Insuasty et al., 2014). A pesar de poder cultivarse en prácticamente todo el país, tanto la producción como el área cultivada se han visto reducidas a partir de 2007, cuando la producción bajó de 107,164 a 70,054 t y el área cultivada de 11,671 a 6,907 ha (Agronet, 2016). Lo anterior puede deberse a que la producción de guayaba en Colombia se basa en la utilización de variedades no mejoradas y de bajo rendimiento y sembradas en traspatio; estas variedades, aunque poco productivas, muestran gran variabilidad genética que debería estudiarse con la posibilidad de explotarse en programas de mejoramiento genético de la especie (Pérez et al., 2016; Rueda et al., 2006).

La provincia de Sumapaz está ubicada en la eco-región central andina colombiana, con climas de frío a cálido, temperaturas entre 2 y $24^{\circ} \mathrm{C}$; alturas desde 480 hasta 3500 msnm y precipitaciones de 1000 a más de 2000 $\mathrm{mm}$ anuales (Jaller, 2010); presenta suelos francos y arcillosos, de moderada profundidad y con buen contenido de materia orgánica, aptos para la agricultura. Las actividades económicas de la población son el turismo y la agricultura, en esta última sobresalen algunos cultivos frutícolas como tomate de árbol (Solanum betaceum Cav.), mora (Rubus glaucus Benth.), uchuva (Physalis peruviana L.), gulupa (Passiflora edulis Sims.) y granadilla (Passiflora ligularis Juss.); en horticultura papa (Solanum tuberosum L.), habichuela (Phaseolus vulgaris L.), arveja (Pisum sativum L.), cebolla (Allium cepa L.), frijol arbustivo (Phaseolus vulgaris L.) y tomate (Solanum lycopersicum Mill.) (Barrientos y Cardona, 2010).

En Sumapaz es común encontrar algunos tipos de guayaba con características morfológicas diferentes, cuyas calidades de fruto no han sido aprovechadas y de las cuales se carece de información. El objetivo de la presente investigación fue caracterizar genotipos silvestres de guayabo a través de descriptores morfológicos y bioquímicos del fruto en los municipios de la provincia del Sumapaz, Cundinamarca, Colombia a fin de conocer la variabilidad genética de la especie en la región y su posible uso en el mejoramiento genético.

\section{MATERIALES Y MÉTODOS}

\section{Área de estudio}

Los genotipos de guayabo se colectaron en la provincia de Sumapaz, región central andina de Colombia (Figura 1). Para la selección de sitios de muestreo se descartaron aquellos lugares con elevaciones superiores a 2000 msnm o por su cercanía al páramo de Sumapaz. Los sitios donde se realizó la colecta de los materiales fueron Silvania, Tibacuy, Pandi, Venecia y Fusagasugá, este último con dos sitios de muestreo: Universidad de Cundinamarca (Udec) y Guavio. Las altitudes de estas localidades oscilaron entre los 1024 y 1765 msnm (Cuadro 1).

\section{Muestreo del material vegetal}

El número de árboles por sitio fue de 10, para un total de 60 árboles analizados. Los árboles se seleccionaron al azar en cada localidad, se dejó una distancia de por lo menos $30 \mathrm{~m}$ entre ellos para evitar recolectar material de árboles emparentados. Los árboles muestreados normalmente se localizaron en zonas de potreros, a orilla de caminos y carreteras y en lugares de vegetación cercana a comunidades. Se cosecharon 20 frutos maduros por árbol, mismos que se utilizaron para la caracterización. Los frutos se lavaron con hipoclorito de sodio al $5 \%$ y se mantuvieron a $4{ }^{\circ} \mathrm{C}$ por un tiempo no mayor de $3 \mathrm{~d}$ para su análisis.

\section{Variables registradas}

Los descriptores morfológicos evaluados se basaron en los propuestos por la Unión Internacional para la Protección de las Obtenciones Vegetales (UPOV, 1987), modificados por Rodríguez-Medina et al. (2010). Se midieron variables cuantitativas y cualitativas del fruto. Las cuantitativas fueron peso del fruto (PF) en g, peso de la pulpa (PP) en g, peso de la cavidad seminal (PCS) en g, diámetro longitudinal (DL) en cm, diámetro transversal (DT) en cm, grosor de la pulpa (GP) en cm y relación peso del fruto/peso de la cavidad seminal. Adicionalmente se determinaron la esfericidad (EF) y porcentaje de pulpa en el peso total del fruto (PT). 


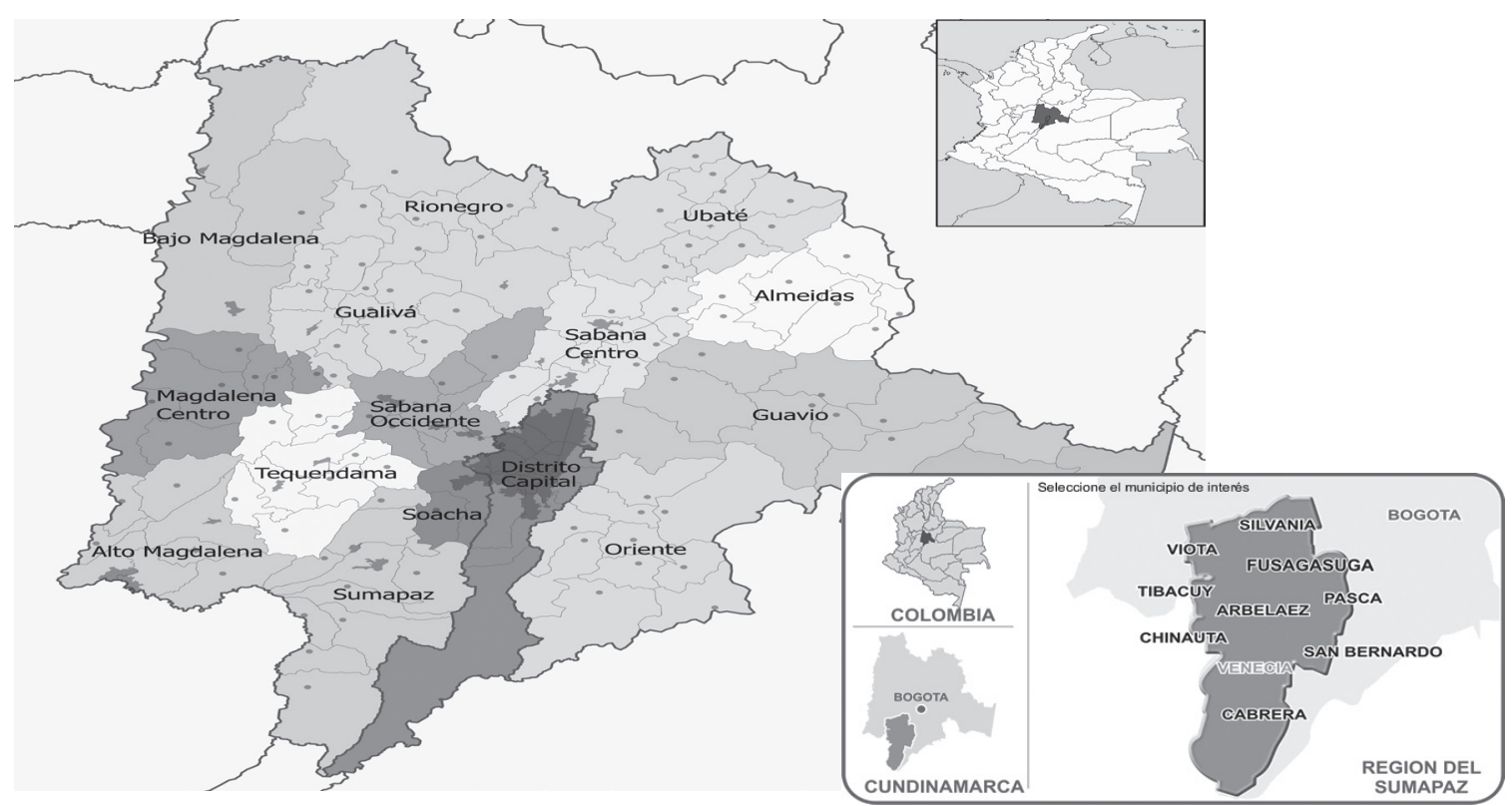

Figura 1. Mapa de Cundinamarca y Sumapaz, Colombia, donde se indican los lugares de muestreo.

Cuadro 1. Características de los sitios de la provincia de Sumapaz donde se colectaron frutos de los árboles de guayabo.

\begin{tabular}{lllcc}
\hline Localidad & Latitud N & Longitud W & Altitud $(\mathrm{msnm})$ & Temperatura media anual $\left({ }^{\circ} \mathrm{C}\right)$ \\
\hline Silvania & $4^{\circ} 24^{\prime} 12^{\prime}$ & $74^{\circ} 23^{\prime} 17^{\prime}$ & 1470 & 23 \\
Tibacuy & $4^{\circ} 20^{\prime} 50^{\prime}$ & $74^{\circ} 27^{\prime} 09^{\prime}$ & 1620 & 24 \\
Pandi & $4^{\circ} 11^{\prime} 28^{\prime}$ & $74^{\circ} 29^{\prime} 16^{\prime}$ & 1024 & 15 \\
Venecia & $4^{\circ} 05^{\prime} 19^{\prime}$ & $74^{\circ} 28^{\prime} 39^{\prime}$ & 1644 & 22 \\
Guavio & $4^{\circ} 18^{\prime} 59^{\prime}$ & $73^{\circ} 38^{\prime} 10^{\prime}$ & 1700 & 19 \\
Udec & $4^{\circ} 20^{\prime} 14^{\prime}$ & $74^{\circ} 21^{\prime} 52^{\prime}$ & 1765 & \\
\hline
\end{tabular}

Para determinar el peso de la cavidad seminal se pesaron frutos individuales, se partieron a la mitad y se separó la pulpa de la semilla, luego se pesó la pulpa y por diferencia con el peso total se obtuvo el valor de la variable.

La esfericidad de los frutos se determinó con base en la relación existente entre los valores de longitud/ancho de los mismos, se consideró que entre más se asemejen estos valores la relación se acerca a 1 y la forma será esférica, los valores se expresaron en porcentaje.

Los caracteres cualitativos fueron color externo y de pulpa, relieve de la superficie, forma del fruto y del pedúnculo. Las determinaciones de color y forma se basaron en las ilustraciones de UPOV (1987) y de Rodríguez-Medina et al. (2010).

\section{Evaluación bioquímica}

Los grados Brix o contenido total de sólidos solubles (SST) se midieron en la pulpa y piel del fruto. Los frutos se maceraron en un mortero hasta obtener una mezcla homogénea y suave que luego se colocó en un refractrómetro digital ATAGO'M 3810 (Bellevue, Washington, USA).

La acidez activa o pH se determinó con un potenciómetro HandyLab 100 (SI Analytics, Mainz, Germany), y para medir la acidez valorable total (AT) se disolvió $1 \mathrm{~g}$ de pulpa en 10 $\mathrm{mL}$ de agua destilada, se usó como titulante $\mathrm{NaOH} 0.5 \mathrm{~N}$ y la fenolftaleína como el indicador (Jiménez et al., 2009). El porcentaje de acidez valorable o titulable se calculó mediante la fórmula:

\footnotetext{
$\%$ Acidez titulable $=\mathrm{N}(\mathrm{NAOH}) \times \mathrm{mL}(\mathrm{NAOH}) \times \mathrm{C}$ Acido $\mathrm{C} \times$ 100/V alícuota
} 
donde: $\mathrm{N}(\mathrm{NAOH})$ es la normalidad del $\mathrm{NaOH}, \mathrm{mL}(\mathrm{NAOH})$ es el volumen de $\mathrm{NaOH}$ consumido en $\mathrm{mL}$; $\mathrm{C}$ Acido $\mathrm{C}$ es igual a $0.64 \mathrm{~g} \mathrm{meq}^{-1}$, que es la constante del ácido cítrico y $\checkmark$ alícuota es el volumen de la muestra.

El protocolo para determinar contenido de vitamina $\mathrm{C}$ se basó en el propuesto por Jagota y Dani (1982) mediante el reactivo Folin-Ciocalteau con algunas modificaciones. Se pesaron $3 \mathrm{~g}$ de fruta y se maceraron con $5 \mathrm{~mL}$ de ácido oxálico $0.15 \%$ durante $3 \mathrm{~min}$. De inmediato se centrifugó durante 120 min a $8500 \mathrm{rpm}$ a $4^{\circ} \mathrm{C}$. Después, se tomaron $800 \mu \mathrm{L}$ del sobrenadante más $1.8 \mathrm{~mL}$ de agua bidestilada y $400 \mu \mathrm{L}$ de Folin-Ciocalteau; posteriormente, se agitó por 20 s y se dejó reposar por 10 min. El blanco utilizado durante la medición fue ácido oxálico $0.15 \%$. La lectura de absorbancia se realizó a 760 nm en un espectrofotómetro Genesys 10S UV-Vis (ThermoFisher Scientific, Waltham, MA USA). Las mediciones se realizaron por triplicado.

Para determinar del contenido de vitamina $\mathrm{C}$ se realizó una curva de calibración con el estándar de AA a 500 ppm en diferentes concentraciones, desde 0.2 hasta $1.2 \mathrm{~mL}$; el procedimiento se replicó seis veces.

\section{Análisis estadístico}

En el caso de vitamina C, los datos se sometieron a análisis de varianza (ANOVA) y de correlación con un criterio de inclusión en el modelo lineal con una $\mathrm{R}^{2}$ superior a 0.9. Se seleccionaron dos modelos lineales, se corrieron los valores de las absorbancias obtenidos en cada ecuación y el promedio de los dos modelos fue el valor reportado en esta investigación.

Los datos del resto de las variables se sometieron a un ANOVA, se usó un diseño completamenteal azar, correlación lineal y prueba de comparación de medias de Tukey $(P \leq$ $0.05)$ con el paquete estadístico Statgraphics Centurion XVII (Statgraphics Technologies Inc., USA). También, con los datos obtenidos para las variables evaluadas se hizo un análisis estadístico básico que incluyó correlaciones con el programa Statgraphics Centurion XVII; además, se elaboró una Matriz Básica de Datos (MBD) y se calculó una matriz de distancias usando el coeficiente DIST con el software estadístico NTSYSpc (Numerical Taxonomic Analysis System) versión 2.2 (Hampl et al., 2001); enseguida, se realizó un agrupamiento no ponderado por conglomerados con las medias aritméticas (UPGMA, Unweighted Pair Group Method Arithmetic Average) y se obtuvo el dendrograma correspondiente.

Adicionalmente, se realizó un análisis de componentes principales para representar la dispersión de los individuos y las relaciones entre variables en los primeros planos factoriales (Hampl et al., 2001). También se realizó una estimación de la variabilidad interna de las muestras poblacionales mediante análisis de componentes principales a través del método propuesto por NúñezColín y Barrientos-Priego (2006). Mediante el cálculo de la distancia euclidiana promedio ponderada de los individuos del grupo k con respecto a su centroide y las gráficas de estos valores con su error estándar se tiene una mejor perspectiva para su interpretación. La medida permite identificar a los individuos atípicos del grupo; es decir, aquellos que presentan datos no comunes; así mismo, ayuda a comparar la dispersión dentro de diferentes grupos de un mismo ACP para determinar la homogeneidad de los individuos.

\section{RESULTADOS Y DISCUSIÓN}

\section{Morfología de fruto}

El ANOVA y la prueba de comparación de medias de Tukey $(P \leq 0.05)$ mostraron la existencia de diferencias significativas entre los sitios de muestreo para todas las características evaluadas (Cuadro 2). El peso de fruto (PF) varió de $45.7 \mathrm{~g}$ en Venecia hasta $120.1 \mathrm{~g}$ en Guavio. Un comportamiento semejante lo mostraron las variables peso de la pulpa (PP), peso de cavidad seminal (PCS) y diámetros del fruto (DL y DT), donde estas mismas colectas mostraron los extremos en estas características; dicha variación se evidencia también en los valores de la desviación estándar (Cuadro 2). El porcentaje de esfericidad (EF) estuvo por encima del 90 \%, lo que indicó predominancia de la forma del fruto tipo circular en cinco de las colectas, con excepción de la de Guavio, que mostró una forma más alargada. Las características grosor de pulpa (GP) y porcentaje de pulpa en el fruto (PT) también mostraron diferencias significativas, donde la de Guavio fue diferente. Los altos valores de variabilidad coinciden con los reportados en el Valle del Cauca por Jiménez et al. (2009), quienes encontraron los mayores porcentajes de variación para las características peso del fruto y de pulpa.

\section{Características bioquímicas}

El ANOVA y la prueba de comparación de medias de Tukey $(P \leq 0.05)$ (Cuadro 3) indicaron que sólo la accesión de Guavio fue significativamente mayor en el pH del resto de las colectas, con un valor en los frutos de 4.68. Valores bajos de pH están relacionados con una mayor vida de anaquel debido a que el pH actúa a nivel fisiológico en el fruto como una barrera natural antimicrobiana (Domene y Segura, 2014). La amplitud de valores de $\mathrm{pH}$ (4.20-4.68) de los genotipos aquí evaluados coincide con los reportados para otras variedades de guayaba (Andrade et al., 2009; Laguado et al., 1999; Suárez et al., 2009), mismos que oscilaron entre 3.7 y 4.7 . 
En lo referente al contenido total de ácidos (ATT), los valores menores correspondieron a frutos de Tibacuy y Udec, con 0.21 y 0.22 , respectivamente, mientras que el valor superior fue para los frutos de Guavio (0.33) (Cuadro $3)$, semejantes a valores previamente reportados para frutos de colectas de la costa colombiana (Castellano et al., 2005), pero distantes de los valores de 0.5 reportados para frutos de la zona de Aguascalientes, México (VázquezOchoa y Colinas-León, 1990) y también diferentes de las guayabas de la India, donde los valores oscilaron entre 3.12 y 6.43 para zonas de cultivo ubicadas a $300 \mathrm{msnm}$ (Dolkar et al., 2017).

La baja acidez en frutos es una ventaja en los mercados internacionales, ya que los ácidos orgánicos logran disminuir el contenido de compuestos volátiles responsables del aroma característico de la guayaba y en esos mercados puede ser un factor de rechazo o aceptación de esta fruta (Mondragón et al., 2009).

En cuanto al contenido de sólidos solubles (SST) medido en grados Brix, se puede observar en el Cuadro 3 que los frutos del genotipo de Guavio presentaron diferencias significativas con respecto al resto, con un valor de 6.46 En la zona occidental del país (Valle del Cauca, Quindío y Risaralda), lugares con alturas desde los 1070 hasta los $1500 \mathrm{msnm}$, se han detectado valores de grados Brix que se ubican entre 2.4 y 12.3 (Jiménez et al., 2009), lo que sugiere que tal característica podría estar asociada con las condiciones climáticas. Los valores de vitamina C (ácido ascórbico) en las colectas oscilaron entre 124.63 y 201.61 $\mathrm{mg} 100 \mathrm{~g}^{-1}$ de fruta fresca (Cuadro 3), que correspondieron a Udec y Pandi, respectivamente, donde las dos mostraron diferencias significativas con respecto a las demás localidades. En Venezuela, las variedades comerciales Criolla Roja y San Miguel mostraron valores de grados Brix

Cuadro 2. Resultados de la prueba de comparación de medias de Tukey $(P \leq 0.05)$ para características morfológicas de frutos de guayaba provenientes de diferentes localidades.

\begin{tabular}{|c|c|c|c|c|c|c|c|c|}
\hline \multirow{2}{*}{ Localidad } & PF & PP & PCS & $D L$ & DT & GP & PT & $\mathrm{EF}$ \\
\hline & \multicolumn{3}{|c|}{ (g) } & \multicolumn{3}{|c|}{$(\mathrm{cm})$} & \multicolumn{2}{|c|}{$(\%)$} \\
\hline Silvania & $68.95 b$ & $42.07 \mathrm{~b}$ & 27.85 bc & $5.04 b$ & $4.87 \mathrm{~b}$ & $0.62 \mathrm{a}$ & $61.38 b$ & $96.94 \mathrm{c}$ \\
\hline Tibacuy & $70.17 b$ & $43.19 b c$ & $26.27 b$ & $5.20 \mathrm{~b}$ & $4.84 b$ & $0.60 \mathrm{a}$ & $61.88 \mathrm{~b}$ & $92.91 \mathrm{~b}$ \\
\hline Pandi & $87.08 \mathrm{c}$ & $53.87 \mathrm{c}$ & $32.31 \mathrm{c}$ & $5.65 c$ & $5.11 \mathrm{~b}$ & $0.64 a$ & 60.35ab & $91.42 \mathrm{~b}$ \\
\hline Venecia & $45.73 \mathrm{a}$ & $27.96 \mathrm{a}$ & $18.78 \mathrm{a}$ & $4.47 \mathrm{a}$ & $4.32 \mathrm{a}$ & $0.57 \mathrm{a}$ & $43.16 \mathrm{a}$ & $96.98 \mathrm{c}$ \\
\hline Guavio & $120.17 d$ & $86.63 d$ & $38.66 \mathrm{~d}$ & $7.48 d$ & $5.92 \mathrm{C}$ & $0.86 \mathrm{~b}$ & $73.69 \mathrm{c}$ & $79.85 a$ \\
\hline Udec & 76.55 & $42.39 b$ & $27.92 \mathrm{bc}$ & $5.02 b$ & $4.83 b$ & $0.56 a$ & 55.31ab & $96.90 \mathrm{c}$ \\
\hline D.S. & 28.74 & 20.63 & 9.93 & 0.85 & 0.69 & 0.17 & 14.31 & 7.76 \\
\hline
\end{tabular}

PF: peso de fruto, PP. peso de la pulpa, PCS: peso de la cavidad seminal, DL: diámetro longitudinal, DT: diámetro transversal, EF: esfericidad, GP. grosor de pulpa, PT: pulpa en fruto, D.S: desviación estándar. Medias con letras iguales en las columnas no son estadísticamente diferentes (Tukey, P $\leq$

Cuadro 3. Comparación de medias (Tukey, $\mathrm{P} \leq 0.05$ ) para características bioquímicas de frutos de guayaba provenientes de diferentes localidades de Colombia.

\begin{tabular}{|c|c|c|c|c|c|}
\hline \multirow{2}{*}{ Localidad } & \multirow{2}{*}{$\mathrm{pH}$} & ATT & SST ('Brix) & SST/ATT & Vitamina C \\
\hline & & \multicolumn{3}{|c|}{$(\%)$} & $\left(\mathrm{mg} 100 \mathrm{~g}^{-1}\right)$ \\
\hline Silvania & $4.21 \mathrm{a}$ & $0.29 a b$ & $9.43 b$ & $34.26 \mathrm{ab}$ & 179.12 bc \\
\hline Tibacuy & $4.22 \mathrm{a}$ & $0.21 \mathrm{a}$ & $9.17 b$ & $46.61 \mathrm{~b}$ & $153.25 \mathrm{ab}$ \\
\hline Pandi & $4.37 \mathrm{a}$ & $0.26 a b$ & $9.41 b$ & $40.42 \mathrm{~b}$ & 124.63 a \\
\hline Venecia & $4.28 a$ & $0.28 a b$ & $9.39 \mathrm{~b}$ & $39.11 \mathrm{~b}$ & 186.23 bc \\
\hline Guavio & $4.68 b$ & $0.33 \mathrm{~b}$ & $6.46 \mathrm{a}$ & 20.98 a & $158.85 \mathrm{abc}$ \\
\hline Udec & $4.20 \mathrm{a}$ & $0.22 \mathrm{a}$ & $8.55 b$ & $39.42 b$ & $201.61 \mathrm{c}$ \\
\hline D.S. & 0.21 & 0.07 & 1.50 & 12.94 & 52.81 \\
\hline
\end{tabular}

pH: potencial de iones H; ATT: contenido total de ácidos; SST: sólidos solubles totales; D.S.: desviación estándar. Medias con letras iguales en las columnas no son estadísticamente diferentes (Tukey, $\mathrm{P} \leq 0.05$ ). 
de 8.5 y 6.8, respectivamente (Laguado et al., 1999). En México, los SST para frutos de Zacatecas se ubicaron entre 12.82 y $11.7^{\circ}$ Brix (Padilla et al., 2014) y en Aguascalientes de 11.9 a 13.2 (Vázquez-Ochoa, y Colinas-León, 1990). En el estado de Pernambuco, Brasil, región semiárida, se reportaron valores desde 9.7 a $13.1^{\circ}$ Brix (Silva et al., 2008).

La mayor zona productora de guayaba en Colombia se encuentra en el estado de Santander, allí se analizó la variedad regional roja, la cual presentó $277.4 \mathrm{mg}$ de ácido ascórbico $100 \mathrm{~g}^{-1}$ de fruta (Olaya y Restrepo, 2012). Para guayabas brasileñas que se siembran también en Santander se reportan valores de 102 mg de ácido ascórbico $100 \mathrm{~g}^{-1}$ de fruta y para guayabas del estado de

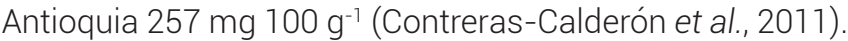
Referencias similares se obtuvieron en India con valores de 104 a 265 mg de ácido ascórbico $100 \mathrm{~g}^{-1}$ de fruta (Dolkar et al., 2017). Comparando con variedades de guayabas mexicanas, se encontró que las de Guanajuato presentaron

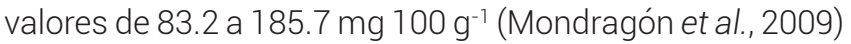
y las de Aguascalientes $163 \mathrm{mg}$ de ácido ascórbico por $100 \mathrm{~g}$ de fruta (Martínez-de Lara et al., 2004; VazquezOchoa y Colinas-Leon, 1990). En Sri Lanka se reportaron $70.4 \mathrm{mg} 100 \mathrm{~g}^{-1}$ (Silva y Sirasa, 2016), lo que indica que se pueden obtener valores desde 70 hasta $277 \mathrm{mg}$ de ácido ascórbico por $100 \mathrm{~g}$ de fruta en frutos de guayaba y que los materiales silvestres de la provincia de Sumapaz presentaron concentraciones dentro de los intervalos reportados, lo que representa un potencial desde el punto de vista nutricional.

La relación de madurez (SST/ATT) permite medir la calidad organoléptica de los frutos (Rodríguez et al., 2006). Valores menores de ATT (ácidos orgánicos) y mayores de SST ('Brix) explican por qué los frutos de guayaba con valores altos de madurez se presentan como frutos más dulces. El contenido de SST se incrementa con la madurez, mientras que compuestos como el almidón y los ácidos disminuyen (Parra-Coronado, 2014). Los frutos de Tibacuy mostraron los valores más altos de SST/ATT, mientras que los más bajos fueron los de Guavio. Se ha reportado que el contenido de azúcares puede ser influenciado por el clima, especialmente por la temperatura del aire y la humedad del suelo que prevalecen en la última etapa de maduración del fruto (Padilla-Ramírez et al., 2016).

Para determinar la variabilidad general mostrada por los caracteres morfológicos y bioquímicos de las colectas de los diferentes sitios se realizó un análisis de todas las variables sin tener en cuenta su origen (Cuadro 4). Los mayores valores de desviación estándar y coeficiente de variación los mostraron el peso de fruto (CV $=50.44 \%$ ), peso de pulpa (CV $=64.58 \%$ ) y peso de cavidad seminal $(\mathrm{CV}=50.12 \%)$. Las características bioquímicas más variables fueron ATT $(\mathrm{CV}=35.09 \%)$ y vitamina C $(\mathrm{CV}=$ $34.59 \%$ ). Estos valores coinciden con los encontrados en el Valle del Cauca, Colombia, donde el peso de la pulpa, del fruto, acidez y la relación grados Brix/acidez presentaron importantes coeficientes de variación (Jiménez et al., 2009).

Se puede apreciar que la forma del fruto que predomina en la zona es redonda, con un contenido de SST que oscila entre 5.1 y $12.8^{\circ}$ Brix y contenido de vitamina $C$ de 61.3 a

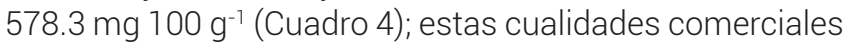
y nutricionales son deseables para un programa de mejoramiento genético en la zona.

\section{Correlaciones entre características}

Se encontró que las características morfológicas de fruto tienen una fuerte relación entre ellas (Cuadro 5), más no las bioquímicas que no mostraron significancia. El valor más alto de correlación $(r=1.0)$ se presentó entre las variables peso de la pulpa y los diámetros longitudinal y transversal del fruto, mientras que el valor más bajo (0.76) fue entre el $\mathrm{pH}$ y el peso de cavidad seminal. El peso de fruto mostró alta correlación positiva con todas las variables referentes a mediciones del fruto como pesos, diámetros y relaciones de estas variables, en tanto la variable esfericidad del fruto tuvo correlaciones altas pero negativas con el resto de las variables (Cuadro 5). En colectas de Tailandia se encontró que las variables morfológicas peso de la fruta, espesor y peso de la pulpa, peso de la cavidad seminal, y químicas como la acidez titulable, pH y ácido ascórbico presentaron correlaciones altas (Thaipong y Boonprakob, 2006). En esta investigación se encontró una relación significativa pero negativa para el $\mathrm{pH}$ y el resto de las variables evaluadas más no para la acidez titulable y el ácido ascórbico. La medición de las propiedades químicas y el tamaño de la fruta combinadas puede usarse para detectar materiales de interés (Thaipong y Boonprakob, 2006).

\section{Análisis de agrupamiento}

Se realizó usando valores de los descriptores morfológicos y bioquímicos. En la Figura 2 se muestra un dendrograma de relaciones entre las colectas evaluadas. Se observa la formación de dos grupos, uno integrado por el genotipo de Guavio y el otro se conformó con el resto de los genotipos. En este segundo grupo es posible diferenciar tres subgrupos, uno con genotipos de las localidades más relacionadas que fueron Tibacuy y Pandi, otro con Silvania y Venecia y el tercer subgrupo corresponde a Udec. Los genotipos de Guavio que forman un grupo aparte, indican que este material vegetal es de particular interés, pues fue sobresaliente en valores de características morfológicas del fruto. 
Cuadro 4. Valores promedio, desviaciones estándar, coeficiente de variación y amplitud de las variables evaluadas en genotipos de guayaba de la provincia de Sumapaz, Colombia.

\begin{tabular}{|c|c|c|c|c|c|}
\hline Característica & Media & D.S. & C.V. & Min & Max \\
\hline Peso de fruto (g) & 78.16 & 39.43 & 50.44 & 16.76 & 226.41 \\
\hline Peso de pulpa (g) & 48.32 & 31.21 & 64.58 & 2.97 & 157.84 \\
\hline Peso de cavidad seminal (g) & 27.22 & 13.64 & 50.12 & 5.28 & 107.11 \\
\hline Diámetro longitudinal (cm) & 5.54 & 1.44 & 26.03 & 2.9 & 10.00 \\
\hline Diámetro transversal (cm) & 4.99 & 0.93 & 18.59 & 2.9 & 7.60 \\
\hline Grosor de pulpa (cm) & 0.65 & 0.22 & 34.22 & 0.3 & 1.30 \\
\hline Pulpa en fruto (\%) & 59.74 & 17.64 & 29.53 & 6.62 & 141.82 \\
\hline Esfericidad del fruto (\%) & 92.08 & 10.46 & 11.36 & 61.05 & 118.75 \\
\hline $\mathrm{pH}$ & 4.31 & 0.32 & 7.32 & 3.7 & 6.4 \\
\hline Acidez del fruto (\%) & 0.32 & 0.11 & 35.09 & 0.1 & 0.6 \\
\hline Sólidos solubles totales ( ${ }^{\circ} \mathrm{Brix}$ ) & 8.62 & 1.78 & 20.66 & 5.1 & 12.8 \\
\hline Relación brix/acidez (\%) & 36.86 & 15.77 & 42.79 & 11.18 & 80.00 \\
\hline Vitamina C (mg 100g-1) & 170.82 & 59.08 & 34.59 & 61.34 & 578.34 \\
\hline
\end{tabular}

D.S.: desviación estándar, C.V.: coeficiente de variación, Min: mínimo, Máx: máximo.

Cuadro 5. Valores de correlación entre las características del fruto de guayaba provenientes de diferentes localidades de la provincia de Sumapaz, Colombia.

\begin{tabular}{lllllllll}
\hline Variable & PF & PP & PCS & DL & DT & GP & PT & EF \\
\hline PF & 1.00 & & & & & & & \\
PP & 0.98 & 1.00 & & & & & & \\
PCS & 0.98 & 0.95 & 1.00 & & & & & \\
DL & 0.96 & 1.00 & 0.92 & 1.00 & & & & \\
DT & 0.99 & 1.00 & 0.97 & 0.98 & 1.00 & & & \\
GP & 0.89 & 0.96 & 0.84 & 0.97 & 0.93 & 1.00 & & \\
PT & 0.88 & 0.95 & 0.86 & 0.94 & 0.94 & 0.95 & 1.00 & 1.00 \\
EF & -0.90 & -0.95 & -0.83 & -0.98 & -0.92 & -0.96 & -0.92 & -0.96 \\
PH & 0.83 & 0.90 & 0.76 & 0.93 & 0.86 & 0.96 & 0.83 & \\
\hline
\end{tabular}

PF: peso de fruto, PP. peso de la pulpa, PCS: peso de cavidad seminal, DL: diámetro longitudinal, DT: diámetro transversal, EF: esfericidad, GP. grosor de la pulpa, PT: pulpa en fruto.

Las características cualitativas no aportaron a la discriminación de los grupos por ser muy homogéneas, por lo que no se involucraron en el análisis de datos.

\section{Análisis de componentes principales}

En el Cuadro 6 se muestran los primeros cinco componentes principales, los cuales explican el $100 \%$ de la variación presente en los genotipos evaluados. El componente principal 1 (CP 1) explica el $74.75 \%$ de la variación y el CP 2 el $12.44 \%$ respecto al total.
En el Cuadro 7 se observó que la mayoría de las variables evaluadas contribuyeron a la conformación del CP 1, con excepción de los grados Brix (SST) y la cantidad de vitamina C. Al CP 2 contribuyó principalmente SST y al CP 3 la variable vitamina $C$.

La dispersión de los genotipos se realizó según los dos primeros componentes principales (Figura 3). Al igual que para el dendrograma (Figura 2), se observó la formación de dos grupos, donde el genotipo menos relacionado fue Guavio, que conformó un grupo y el resto de las colectas integraron al segundo grupo. Los genotipos de Guavio 


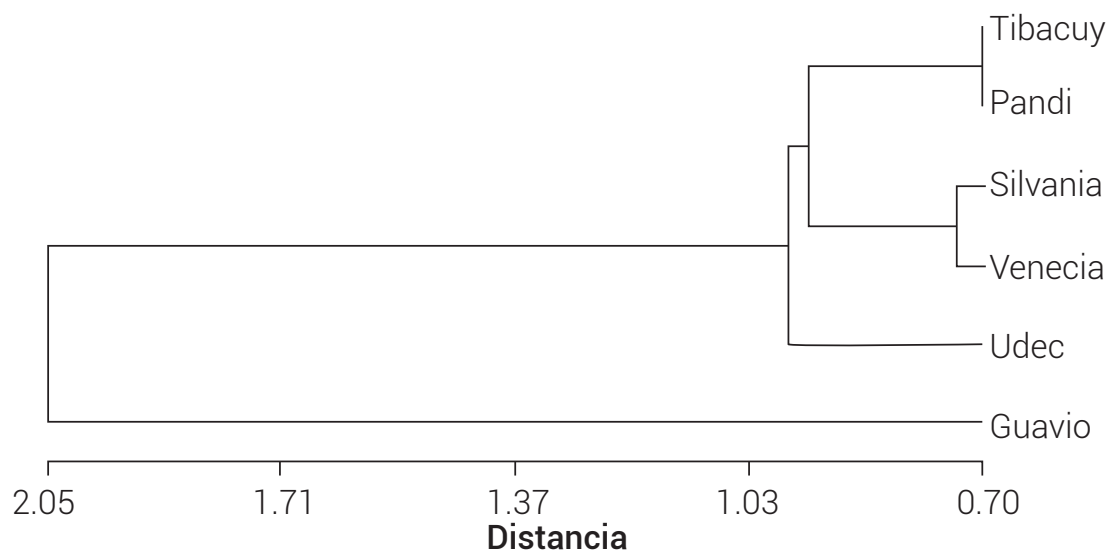

Figura 2. Dendrograma de relaciones entre las colectas de guayaba evaluadas, procedentes de diferentes sitios de muestreo de la provincia de Sumapaz, Colombia, construido con el método UPGMA.

Cuadro 6. Valores propios, porcentaje de variación explicada por cada componente y porcentaje de varianza acumulada para cinco componentes principales (CP).

\begin{tabular}{lccc}
\hline CP & Valores propios & Porcentaje de varianza explicada & Porcentaje de varianza acumulada \\
\hline 1 & 9.72 & 74.75 & 74.75 \\
2 & 1.62 & 12.44 & 87.19 \\
3 & 1.22 & 9.39 & 96.58 \\
4 & 0.25 & 1.92 & 98.50 \\
5 & 0.19 & 1.50 & 100.00 \\
\hline
\end{tabular}

Cuadro 7. Contribución de las variables morfológicas y bioquímicas de frutos de guayaba a los vectores propios de los cuatro primeros componentes principales (CP).

\begin{tabular}{|c|c|c|c|c|}
\hline Variable original & CP 1 & CP 2 & $\mathrm{CP} 3$ & CP 4 \\
\hline PF & 0.945 & 0.304 & 0.036 & 0.081 \\
\hline PP & 0.986 & 0.161 & 0.021 & 0.02 \\
\hline PCS & 0.913 & 0.308 & -0.040 & 0.244 \\
\hline DL & 0.991 & 0.111 & 0.015 & -0.065 \\
\hline DT & 0.974 & 0.203 & 0.017 & 0.100 \\
\hline GP & 0.988 & -0.108 & 0.042 & -0.069 \\
\hline PT & 0.945 & 0.03 & -0.079 & 0.079 \\
\hline EF & -0.963 & -0.055 & 0.078 & 0.245 \\
\hline $\mathrm{pH}$ & 0.94 & -0.146 & 0.012 & -0.281 \\
\hline AT & 0.696 & -0.66 & 0.219 & 0.082 \\
\hline SST & -0.202 & 0.874 & 0.422 & -0.088 \\
\hline STT/AT & -0.834 & 0.328 & -0.435 & -0.072 \\
\hline Vitamina C & -0.431 & -0.085 & 0.887 & -0.015 \\
\hline
\end{tabular}

CP. componentes principales, PF: peso de fruto, PP. peso de la pulpa, PCS: peso de cavidad seminal, DL: diámetro longitudinal, DT: diámetro transversal, EF: esfericidad, GP. grosor de pulpa, PT: pulpa en fruto, pH: potencial de iones H, AT: contenido total de ácidos, SST: sólidos solubles. 


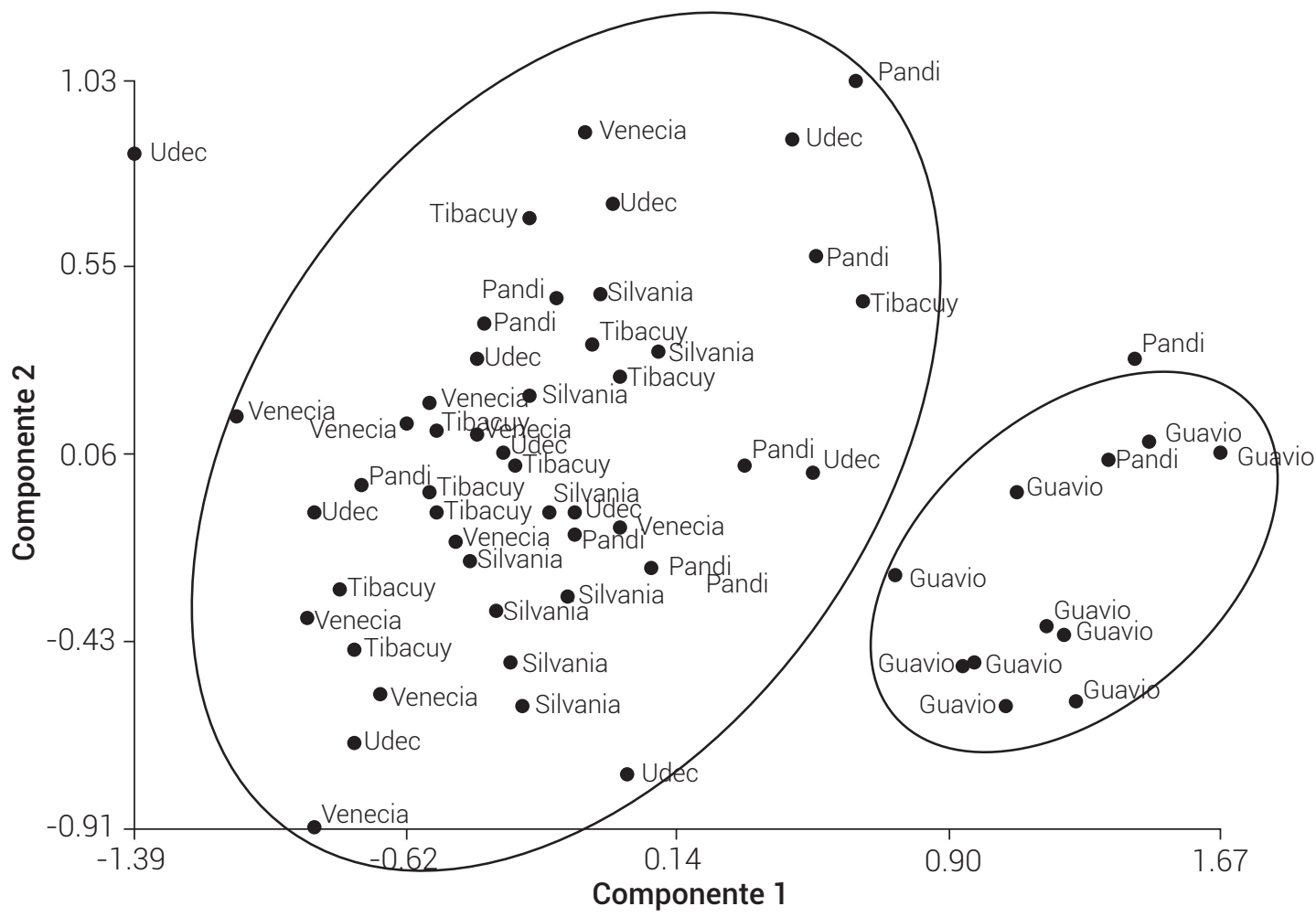

Figura 3. Diagrama en 2D que muestra las relaciones entre las colectas de guayaba procedentes de diferentes sitios de muestreo de la provincia de Sumapaz, Colombia.

son muy diferentes del resto, posiblemente, debido a la distancia geográfica mayor que existe entre esta localidad y las otras, o a las barreras geográficas que dificultan el flujo genético (Figura 1).

En la Figura 4 se muestra la variabilidad intragrupo detectada en frutos de guayaba procedentes de diferentes sitios de muestreo de la provincia de Sumapaz, Colombia. La varianza interna ponderada es la distancia euclidiana promedio al cuadrado de los individuos con respecto a su centroide \pm error estándar. Se observó que los frutos más homogéneos son los de Guavio, Silvania y Tibacuy, mientras que los más heterogéneos son los de Venecia, Pandi y Udec. Por el alto error estándar que presentan las colectas de Pandi, Udec y Venecia se puede considerar que existen algunos individuos atípicos dentro de ellas, por lo que se genera una alta variabilidad intragrupo. Las colectas de Guavio fueron las que mostraron tanto la menor varianza interna como el error estándar más bajo, lo que sugiere que en ese caso no existen individuos atípicos o hay mayor homogeneidad.

\section{CONCLUSIONES}

Los árboles de guayabo evaluados en la provincia de Sumapaz presentan características de interés comercial y nutricional que podrían servir como base para establecer un programa de mejoramiento en la zona. El peso del fruto fue el carácter que presentó mayor variabilidad, por lo que es la medida más importante y explica el comportamiento del resto de variables en lo referente a la morfología del fruto. Dentro de las colectas se encontró al tipo Guavio como sobresaliente por sus características morfológicas y diferente del resto, aunque sus contenidos de SST o vitamina C fueron bajos. En el análisis de agrupamiento se encontró que Guavio conformó un solo grupo y las demás colectas otro. Las colectas de Guavio fueron las que mostraron tanto la menor varianza interna como el error estándar más bajo.

\section{AGRADECIMIENTOS}

A Heliana Torres Opayome, Alberto Suarez Quiñonez, Jeison David Montoya, Yimy Leonardo Villalobos Gutiérrez y Humberto Morales, laboratoristas del Laboratorio de Química, Microbiología, Suelos y Fisiología de la Universidad de Cundinamarca sede Fusagasugá. 


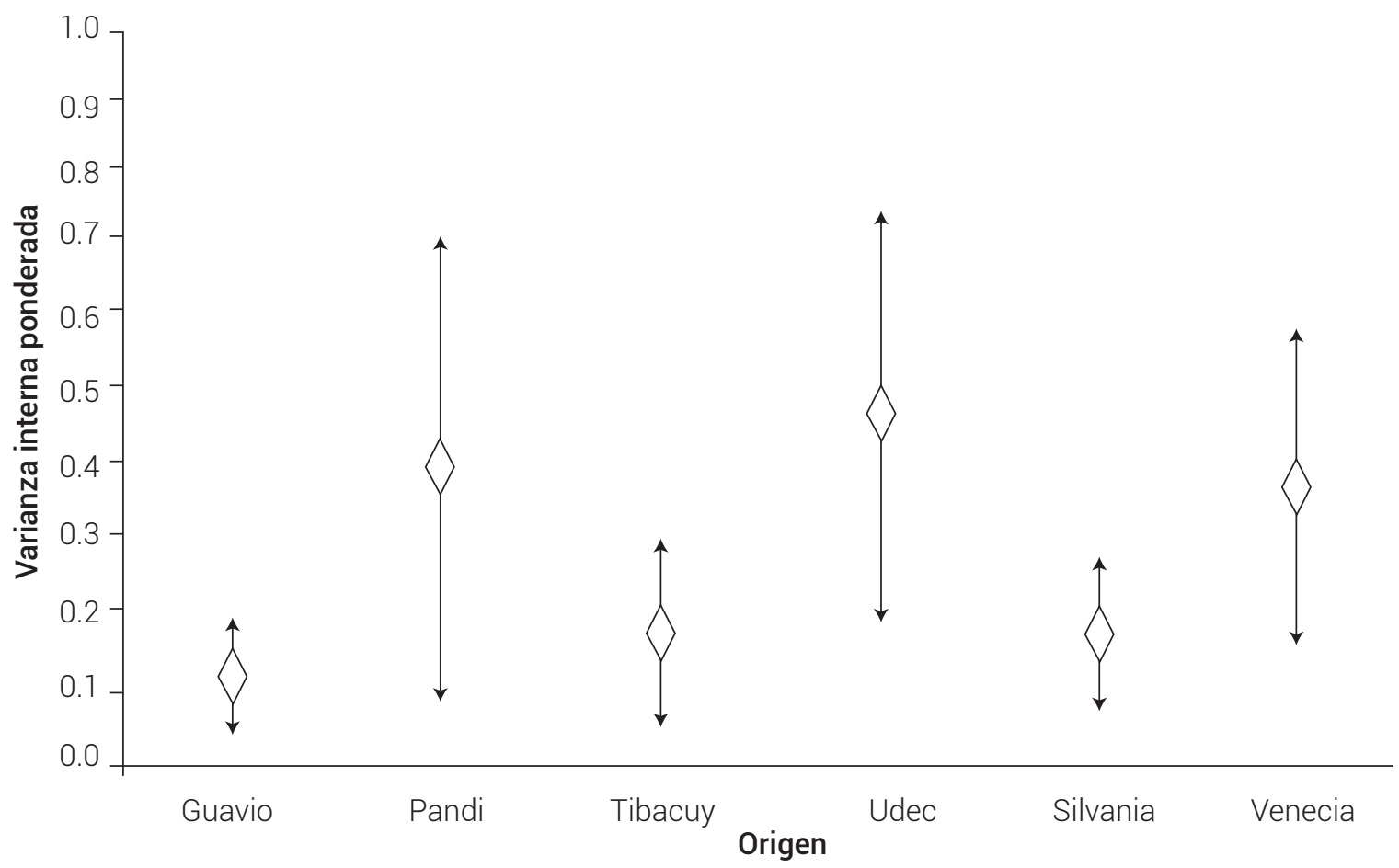

Figura 4. Variabilidad intragrupo de frutos de guayaba procedentes de diferentes sitios de muestreo de la provincia de Sumapaz.

\section{BIBLIOGRAFÍA}

Agronet, MinAgricultura (2016) Estadísticas agropecuarias. Red de información y Comunicación del Sector Agropecuario Colombiano. Bogotá. Colombia. http://www.agronet.gov.co/ estadistica/Paginas/default.aspx (Marzo 2018).

Andrade P. R. D., F. A. Ortega Q., E. J. Montes M., R. Torres G., O. A. Pérez S., M. Castro N. y L. A. Gutiérrez S. (2009) Caracterización fisicoquímica y reológica de la pulpa de guayaba (Psidium guajava L.) variedades híbrido de Klom Sali, Puerto Rico, D14 y Red. Vitae 16:13-18.

Barrientos F. J. C. y J. O. Cardona (2010) Los pequeños productores están limitados para adoptar nuevos cultivos. El caso de las hierbas aromáticas en la región de Sumapaz, Cundinamarca. Agronomía Colombiana 28:99-106.

Castellano G., O. Quijada, R. Ramírez y E. Sayago (2005) Comportamiento poscosecha de frutas de guayaba (Psidium guajava L.) tratados con cloruro de calcio y aqua caliente a dos temperaturas de almacenamiento. Revista Iberoamericana de Tecnología Postcosecha 6:78-82.

Contreras-Calderón J., L. Calderón-Jaimes, E. Guerra-Hernández and B. García-Villanova (2011) Antioxidant capacity, phenolic content and vitamin $\mathrm{C}$ in pulp, peel and seed from 24 exotic fruits from Colombia. Food Research International 44:2047-2053, https://doi.org/10.1016/j.foodres.2010.11.003

Dolkar D., P. Bakshi, M. Gupta, V. K. Wali, R. Kumar, T. K. Hazarika and D. Kher (2017) Biochemical changes in guava (Psidium guajava) fruits during different stages of ripening. Indian Journal of Agricultural Sciences 87:257-260.

Domene R. M. A. y M. Segura R. (2014) Parámetros de calidad interna de hortalizas y frutas en la industria agroalimentaria. Ficha de Transferencia 5. Grupo Cooperativo Cajamar. Almería, España. $18 \mathrm{p}$.

Hampl V., A. Pavliěk and J. Flegr (2001) Construction and bootstrap analysis of DNA fingerprinting-based phylogenetic trees with the freeware program FreeTree: application to trichomonad parasites.
International Journal of Systematic and Evolutionary Microbiology 51:731-735, https://doi.org/10.1099/00207713-51-3-731

Insuasty B. 0., R. Monroy R., A. Díaz F. y J. Bautista D. (2014) Manejo Fitosanitario del Cultivo de la Guayaba (Psidium guajava L.) en Santander. Corporación Colombiana de Investigación Agropecuaria, Instituto Colombiano Agropecuario. Bogotá Colombia. $40 \mathrm{p}$.

JagotaS. K. and H. M. Dani(1982) A new colorimetric technique for the estimation of vitamin C using Folin phenol reagent. Analytical Biochemistry 127:178-182, https://doi.org/10.1016/0003-2697(82)90162-2

Jaiswal U. and V.S. Jaiswal (2005) Psidium guajava guava. In: Biotechnology of Fruit and Nut Crops. R.E. Litz (ed.). Cabi Publishing. Wallingford, UK. pp:394-402.

Jaller R. S. (2010) Análisis de los Sistemas de Producción Agrícola de las Provincias de Soacha y Sumapaz (Cundinamarca) Organización de las Naciones Unidas para la Agricultura y la Alimentación. Bogotá, Colombia. 210 p.

Jiménez L. L., M. I. Almanza P. y J. E. Muñoz F. (2009) Caracterización morfológica de accesiones silvestres de guayaba. Acta Agronómica 58:69-73.

Laguado N., E. Pérez, C. Alvarado y M. Marín (1999) Características fisicoquímicas y fisiológicas de frutos de guayaba de los tipos Criolla Roja y San Miguel procedentes de dos plantaciones comerciales. Revista de la Facultad de Agronomía - LUZ 16:382-397.

Martínez-De Lara J., M. C. Barrientos-Lara, A. C. Reyes-De Anda, S Hernández-Delgado, J. S. Padilla-Ramírez y N. Mayek P. (2004) Diversidad fenotípica y genética en huertas de guayabo de Calvillo, Aguascalientes. Revista Fitotecnia Mexicana 27:243249.

Mata B. I. y A. Rodríguez M. (1990) Cultivo y Producción del Guayabo. Trillas. México, D. F. 160 p.

Mondragón J. C., L. M. Toriza A. y S. H. Guzmán M. (2009) Caracterización de selecciones de guayaba para el Bajío de Guanajuato, México. Agricultura Técnica en México 35:315-322.

Núñez-Colín C. A. y A. F. Barrientos-Priego (2006) Estimación de la variabilidad interna de muestras poblacionales, mediante 
análisis de componentes principales. Interciencia 31:802-806.

Olaya Z. J. A. y L. P. Restrepo S. (2012) Estudio del contenido de fenoles y actividad antioxidante de guayaba en diferentes estados de madurez. Acta Biológica Colombiana 17:611-624.

Padilla R. J. S., E. González G., V. M. Rodríguez M., L. Reyes M., E. S. Osuna C. y E. Acosta D. (2014) Varianza entre y dentro e índice de repetitividad de características cuantitativas de fruto de guayaba. Revista Mexicana de Ciencias Agrícolas 5:1423-1432.

Padilla-Ramírez J. S., V. M. Rodríguez-Moreno, E. González-Gaona, C. J. Cortes-Penagos y E. Acosta-Díaz (2016) Caracterización morfológica de fruto de la colección ex situ de Psidium guajava L. Agroproductividad 9:9-18.

Parra-Coronado A. (2014) Maduración y comportamiento poscosecha de la guayaba (Psidium guajava L.). Una revisión. Revista Colombiana de Ciencias Hortícolas 8:314-327.

Pérez P. L., A. Sigarroa G., E. Bandera F., N. N. Rodríguez M., M. T. Cornide and J. E. Sánchez G. (2016) Characterization of genetic diversity in three guava (Psidium guajava L.) populations. Cultivos Tropicales 37:115-126.

Rodríguez N. N., J. Valdés, J. A. Rodríguez, J. B. Velásquez, D. Rivero, F. Martínez, G. González, D. G. Sourd, L. González and J. Cañizares (2010) Genetic resources and breeding of guava (Psidium guajava L.) in Cuba. Biotecnología Aplicada 27:238-241.

Rodríguez M., H. E. Arjona y J. A. Galvis (2006) Maduración del fruto de feijoa (Acca sellowiana Berg.) en los clones 41 (Quimba) y 8-4 a temperatura ambiente en condiciones de la Sabana de Bogotá Agronomía Colombiana 24:68-76.

Rodríguez-Medina N. N., G. A. Fermin, J. Valdés-Infante, B. Velásquez, D. Rivero, F. Martínez, J. Rodríguez and W. Rohde (2010) Illustrated descriptors for guava (Psidium guajava). Acta Horticulturae 849:103-110, https://doi.org/10.17660/ActaHortic.2010.849.11
Rueda A., J. D. Palacio, J. E. Muñoz, R. Saavedra y E. Bravo (2006) Caracterización molecular del banco de germoplasma de guayaba Psidium spp. del Centro de Investigación CorpoicaPalmira. Fitotecnia Colombiana 6:26-32.

Silva J. J. F., J. E. F. Bezerra, J. A. Tavares, I. E. Lederman y M. L. M. Neto (2008) Caracterización y evaluación de germoplasma de guayabo (Psidium guajava L.) en la región semiárida del estado de Pernambuco, Brasil. Revista Caatinga 21:94-99.

Silva K. D. R. R. and M. S. F. Sirasa (2018) Antioxidant properties of selected fruit cultivars grown in Sri Lanka. Food Chemistry 238:203-208, https://doi.org/10.1016/j.foodchem.2016.08.102

Suárez J., M. Pérez C y A. Giménez (2009) Efecto de la temperatura y estado de madurez sobre la calidad poscosecha de la fruta de guayaba (Psidium guajava L.) procedente de Mercabar, estado Lara, Venezuela. Revista UDO Agrícola 9:60-69.

Thaipong K. and U. Boonprakob (2006) Repeatability, optimal sample size of measurement and phenotypic correlations of quantitative traits in guava. Kasetsart Journal (Natural Science) 40:11-19.

UPOV, International Union for the Protection of New Varieties of Plants (1987) Guidelines for the conduct of tests for distinctness, homogeneity and stability. Guava (Psidium guajava L.) TG/110/3. International Union for the Protection of New Varieties of Plants. Geneve, Switzerland. 27 p.

Vazquez-Ochoa R. I. y M. T. Colinas-Leon (1990) Changes in guavas of three maturity stages in response to temperature and relative humidity. HortScience 25:86-87.

Yam T. J. A., C. A. Villaseñor P., E. Romantchik K., M. Soto E. y M. A. Peña P. (2010) Una revisión sobre la importancia del fruto de guayaba (Psidium guajava L.) y sus principales características en la postcosecha. Revista Ciencias Técnicas Agropecuarias 19:7482. 
